\title{
Prevalence of chronic kidney disease defined by using CKD-EPI equation and albumin-to-creati- nine ratio in the Korean adult population
}

Eunhee $\mathrm{Ji}^{1,2}$ and Yon $\mathrm{Su} \mathrm{Kim}^{3}$

${ }^{1}$ College of Pharmacy and Institute of Pharmaceutical Sciences, Gachon University, Incheon; ${ }^{2}$ Clinical Trials Center, Gachon University Gil Medical Center, Incheon; ${ }^{3}$ Department of Internal Medicine, Seoul National University College of Medicine, Seoul, Korea
Received: June 29, 2015

Revised : July 31, 2015

Accepted: August 13, 2015

\section{Correspondence to}

Yon Su Kim, M.D.

Department of Internal Medicine, Seoul National University College of Medicine, 103 Daehak-ro,

Jongno-gu, Seoul 03080, Korea

Tel: $+82-2-2072-2264$

Fax: +82-2-745-2264

E-mail: yonsukim@snu.ac.kr
Background/Aims: An updated chronic kidney disease (CKD) definition and classification were proposed by Kidney Disease: Improving Global Outcomes (KDIGO), with adoption of a new equation to estimate glomerular filtration rate (GFR) and albuminuria to evaluate kidney structural damage. This study was performed to estimate the prevalence of CKD in the Korean adult population as defined and classified by the KDIGO guidelines.

Methods: Cross-sectional samples of the fifth Korean National Health and Nutrition Examination Survey for 2011 to 2012 were examined for adults aged $\geq 19$ years. CKD prevalence was determined based on decreased GFR and albuminuria. The GFR was estimated using the CKD Epidemiology Collaboration creatinine equation, and albuminuria was evaluated using the albumin-to-creatinine ratio (ACR) in spot urine.

Results: Of the 16,576 subjects participating in the survey, 10,636 (4,758 men, 5,878 women) were included in the present study. The prevalence of CKD was estimated as $7.9 \%(7.8 \%$ in 2011 and $8.0 \%$ in $2012, p=0.770)$. The prevalence of low, moderately increased, high, and very high CKD risk prognosis was 92.0\%, 6.3\%, $1.1 \%$, and $0.6 \%$, respectively. The prevalence of albuminuria (ACR $\geq 30 \mathrm{mg} / \mathrm{g}$ ) in individuals with GFR $\geq 60 \mathrm{~mL} / \mathrm{min} / 1.73 \mathrm{~m}^{2}$ has reached $5.7 \%$. The odds ratios of hypertension and diabetes to CKD were 3.4 and 3.1 in men, and 2.9 and 2.0 in women (all $p<0.001)$, respectively.

Conclusions: A large percentage of CKD patients had albuminuria prior to a decrease in GFR. Regular laboratory tests for albuminuria for the high-risk group, and especially for hypertensive or diabetic patients, might improve detection of CKD at an early stage.

Keywords: Renal insufficiency, chronic; Kidney Disease Improving Global Outcomes; Glomerular filtration rate; Albuminuria; Korea

\section{INTRODUCTION}

Chronic kidney disease (CKD) is defined as a structural or functional kidney abnormality lasting for 3 or more months [1]. The global prevalence of CKD is estimated to be more than $10 \%$, and CKD has emerged as a public health problem [2]. Adverse outcomes of CKD such as kidney failure, cardiovascular disease (CVD), and premature death can be prevented or delayed when treatment is initiated in the early stages of disease [3]. As the earlier 
stages are often asymptomatic, CKD is usually detected during laboratory evaluation of comorbid conditions.

Glomerular filtration rate (GFR) is considered the index of kidney function, and for convenience is usually estimated using equations instead of by direct measurement. The Modification of Diet in Renal Disease (MDRD) Study equation [4], which was replaced by the Chronic Kidney Disease Epidemiology Collaboration 2009 equations (CKD-EPI equations) [5], has been used to estimate GFR. However, this tended to underestimate the measured GFR and had less precision, especially at a GFR of $60 \mathrm{~mL} / \mathrm{min} / 1.73 \mathrm{~m}^{2}$ or more [6]. The CKD-EPI equations were reported as having less bias, improved precision, and greater accuracy than the MDRD Study equation at a higher GFR of $60 \mathrm{~mL} / \mathrm{min} / 1.73 \mathrm{~m}^{2}$ or more [5]. This was also demonstrated for the Korean population [7]. The CKD-EPI equations led to a lower estimation of CKD prevalence, primarily due to a lower prevalence of GFR stage 3, compared to the MDRD Study equation [5]. Persistently increased protein excretion has usually been considered to be a marker of kidney damage, and screening with urine dipsticks has been acceptable for detecting proteinuria [1]. Recently, urinary albumin measurement was recommended as a marker of kidney damage, both for its standardization and also because albumin is the most important protein lost in the urine in most CKD [8]. The albumin-to-creatinine ratio (ACR) is the most preferred value among urinary albumin measurements. Kidney Disease: Improving Global Outcomes (KDIGO) has updated guidelines for defining, diagnosing, staging, and managing CKD since the establishment of the first uniform definitions and staging system for CKD in 2002 [8]. More objective criteria, such as the CKD-EPI equations for estimating GFR and classification of albuminuria, have been incorporated. Moreover, GFR stage 3 was subdivided into 3 a and $3 \mathrm{~b}$. The etiology of CKD was included in the definition; hypertension and diabetes mellitus (DM) are systemic diseases known to cause CKD. Consequently, KDIGO guidelines provide relatively simple and precise methods for early diagnosis of CKD, and tend to yield fewer false positives, thereby avoiding incorrect diagnosis [9].

The number of patients treated with renal replacement therapy (RRT) including dialysis and transplantation has increased dramatically in Korea, from 28,046 in 2000 to 75,042 in 2013 [10]. The prevalence of RRT was
1,446 patients per million population ( $\mathrm{pmp}$ ), with an incidence of $234 \mathrm{pmp}$ in 2013. The cause of kidney failure was estimated to be DM in $48.0 \%$, hypertension in $19.7 \%$, and chronic glomerulonephritis in $8.3 \%$ of cases. The previously reported CKD prevalence calculated from the MDRD Study equation together with ACR was $13.7 \%$ in urban Korean volunteers aged $\geq 35$ [11]. The CKD prevalence calculated from the MDRD Study equation alone was $8.8 \%$ in 2005 and $7.2 \%$ in 2007 in Korea National Health and Nutrition Examination Survey (KNHANES) subjects aged $\geq 20$ [12]; the prevalence calculated using the MDRD Study equation with the dipstick proteinuria method has tended to decrease since 2001 in men and since 2005 in women among KNHANES I to IV (1998 to 2009) subjects aged $\geq 20$ [13]. Since there are no reports on CKD prevalence using the CKD-EPI equations to estimate GFR and ACR to measure albuminuria for Koreans, a new analysis of CKD prevalence is necessary. This study was therefore designed to investigate the prevalence of CKD in the Korean population using KNHANES samples, based on the definition and classification by KDIGO 2012 guidelines, as well as the impact of changes through a comparison of the CKD-EPI and MDRD Study equations for the estimation of GFR, and the associations between CKD and major risk factors, including DM, hypertension, age, and gender.

\section{METHODS}

\section{Study population}

This retrospective, population-based study was performed using data acquired by the KNHANES $\mathrm{V}$ from 2010 to 2012 [14]. KNHANES V was a cross-sectional, nationally representative survey with a multistage, stratified, and probability sampling design based on sex, age, and geographical area using household registries. KNHANES, composed of a health questionnaire survey, physical examination, and nutrition survey, has been conducted periodically since 1998 to assess the health and nutritional status of the non-institutionalized Korean population by the Korea Centers for Disease Control and Prevention (KCDC). The study protocol was approved by the Institutional Review Board of KCDC (2010-02CON-21-C), and written informed consents were obtained from all subjects or their guardians. KN- 
HANES V data of 2010 were excluded as urine albumin was not measured until 2011. The inclusion criteria were (1) adults aged $\geq 19$, and (2) available serum creatinine (Scr), urine albumin, and urine creatinine test results. Individuals aged $\geq 19$ were classified as adults in the study due to the KDIGO guideline's classification of children as birth to 18 [8].

\section{Anthropometry and laboratory data}

The health examination was performed in a mobile health examination center. All procedures were performed under quality control. Blood pressure (BP) was measured by a trained nurse following a standard protocol in accordance with the recommendations of the American Heart Association. Three readings were obtained via mercury sphygmomanometer (Baumanometer, Baum, Copiague, NY, USA), and the mean of the second and third reading was reported as the final BP for individual participants. Body mass index (BMI) was calculated as body weight divided by the square of the height, and expressed in units of $\mathrm{kg} / \mathrm{m}^{2}$.

Blood samples were collected from the median cubital or cephalic veins by a skilled nurse after an overnight fast. A random urine sample (first urine in the morning, if possible) was obtained in a conical container. The urine and pretreated blood samples were refrigerated and transported to the central laboratory within the day (Neodin Medical Institute, Seoul, Korea). Serum creatinine and urine creatinine were assayed by colorimetry using Creatinine-HR L-type Wako (Wako, Osaka, Japan) following the Jaffe method; fasting glucose concentration (fasting blood glucose, FBG) by an enzymatic method using Pureauto S GLU (Sekisui, Osaka, Japan); total cholesterol, high density lipoprotein cholesterol (HDL-C), and triglycerides by an enzymatic method; and urine albumin by turbidimetric assay using Albumin (Roche, Mannheim, Germany), all performed using a Hitachi Automatic Analyzer 7600 (Hitachi High-Technologies Co., Tokyo, Japan), which was calibrated using Calibrator for Automated Systems (Roche Diagnostics, Indianapolis, IN, USA). Glycated hemoglobin (Aıc) was assayed by high performance liquid chromatography (HLC-723G7 A1c, Tosoh, Tokyo, Japan). Urine chemistry was tested by test strip (Urysis 2400 cassette, Roche) using Urisys 2400 (Roche). Low density lipoprotein cholesterol (LDL-C) was computed using the Friedewald formula when triglycerides were less than $400 \mathrm{mg} / \mathrm{dL}$.

\section{Definitions of CKD and other chronic diseases}

CKD was defined and classified based on KDIGO guidelines using both GFR and urinary ACR [8]. GFR was estimated using CKD-EPI equations, taking into account gender and serum creatinine. ACR was computed and reported in milligrams per gram. Individuals with GFR $<60 \mathrm{~mL} / \mathrm{min} / 1.73 \mathrm{~m}^{2}$ or ACR $\geq 30 \mathrm{mg} / \mathrm{g}$ were classified as CKD patients. Hypertension was defined as having a systolic blood pressure (SBP) of $\geq 140 \mathrm{mmHg}$ or a diastolic blood pressure (DBP) of $\geq 90 \mathrm{mmHg}$, or having received a physician's diagnosis. DM was defined as having Alc $\geq 6.5 \%$, FBG of $\geq 126 \mathrm{mg} / \mathrm{dL}$, or having received a physician's diagnosis. Dyslipidemia was defined as having total cholesterol $\geq 240 \mathrm{mg} / \mathrm{dL}, \mathrm{LDL}-\mathrm{C} \geq 160 \mathrm{mg} / \mathrm{dL}$, HDL-C $<40 \mathrm{mg} / \mathrm{dL}$, triglycerides $\geq 200 \mathrm{mg} / \mathrm{dL}$, or having received a physician's diagnosis.

\section{Statistical analysis}

Analyses were performed incorporating the sampling weights to obtain unbiased estimates from the complex KNHANES sampling design following the KNHANES recommendations. The study population was separated as a subpopulation, and the analysis was carried out on the entire KNHANES samples for 2011 and 2012 to represent all Korean adults without bias. In addition, weight adjustments were applied to the user-missing values to prevent skews in sampling design. The demographic and biochemical characteristics of the study population were summarized and compared using the complex samples general linear model for continuous variables and the complex samples chi-square test for categorical variables. The mean and the $95 \%$ confidence interval (CI) were presented for data on continuous variables, whereas unweighted count with weighted percentage was presented for data on categorical variables. The complex samples chi-square test was used to analyze prevalence of CKD by GFR with ACR categories, and GFR comparison between MDRD and CKD-EPI equations was expressed as a weighted percentage. A subpopulation was added to strata when performing the complex samples chi-square test. Associations of CKD, GFR, and ACR categories with various risk factors, including gender, age, BMI, DM, hypertension, and dyslipidemia were expressed as an odds ratio (OR), which 
was calculated using complex samples multivariate logistic regression analyses. All statistical analyses were performed using IBM SPSS version 21.0 (IBM Corp., Armonk, NY, USA). The statistical significance level was set at $p<0.05$.

\section{RESULTS}

\section{Characteristics of the study population}

Among the total sample size of 16,576 from 2011 and 2012, 64.7\% satisfied the inclusion criteria (10,636 individuals: 5,388 and 5,248 from 2011 and 2012, respectively) as shown in Fig. 1. The composition of neither the total adults nor the study population was statistically different between 2011 and $2012(p=0.232$ and $p=0.087$, respectively). The study population comprised $81.8 \%$ of total adults (81.0\% and 82.7\% from 2011 and 2012, respectively, $p=0.173)$. The anthropometric and biochemical characteristics of the study population in 2011 and 2012 were comparable, except for HDL-C, LDL-C, and prevalence of dyslipidemia (Table 1). The percentage of men was $52.6 \%$, and the mean age was 45.8 years ( $95 \% \mathrm{CI}$, 45.2 to 46.3$)$. The prevalence of BMI $\geq 25$ was $33.2 \%$. Mean serum creatinine was $0.86 \mathrm{mg} / \mathrm{dL}$ (95\% CI, 0.85 to 0.86 ), with an estimated GFR of $96 \mathrm{~mL} / \mathrm{min} / 1.73 \mathrm{~m}^{2}$ (95\% CI, 96 to 97). Mean ACR was $18 \mathrm{mg} / \mathrm{g}$ (95\% CI, 15 to 21 ), with urine albumin $22 \mu \mathrm{g} / \mathrm{mL}$ (95\% CI, 19 to 26 ) and urine creatinine $165 \mathrm{mg} / \mathrm{dL}$ (95\% CI, 163 to 168). Mean SBP was $118 \mathrm{mmHg}$ (95\% CI, 117 to 119 ), and DBP $76 \mathrm{mmHg}$ (95\% CI, 76 to 77). Mean FBG was $97 \mathrm{mg} / \mathrm{dL}$ (95\% CI, 97 to 98 ), and Aic 5.7\% (95\% CI, 5.67 to 5.71). Mean total cholesterol,

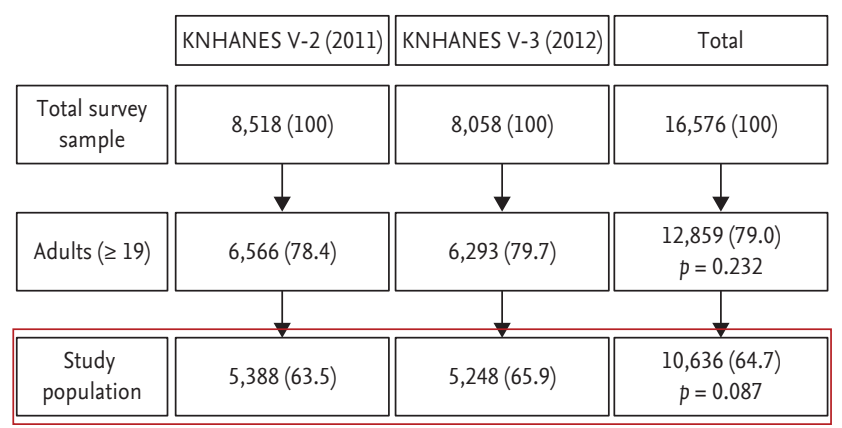

Figure 1. Selection of study population from the total survey samples. Values are presented as number (\%). KNHANES V, the Fifth Korea National Health and Nutrition Examination Survey.
HDL-C, LDL-C, and triglycerides were $190 \mathrm{mg} / \mathrm{dL}$ ( $95 \%$ CI, 188 to 191 ), $49 \mathrm{mg} / \mathrm{dL}$ (95\% CI, 49 to 50 ), $114 \mathrm{mg} / \mathrm{dL}$ ( $95 \% \mathrm{CI}, 113$ to 115 ), and $136 \mathrm{mg} / \mathrm{dL}$ (95\% CI, 133 to 139 ), respectively.

\section{Prevalence of CKD and other chronic diseases}

The CKD prevalence in Korean adults was estimated as $7.9 \%$ (7.8\% in 2011 and 8.0\% in 2012, $p=0.770$ ) (Table 1). The prevalence of hypertension, DM, and dyslipidemia was $27.8 \%$ (27.2\% in 2011 and $28.4 \%$ in $2012, p=0.380$ ), $9.3 \%$ (9.7\% in 2011 and $9.0 \%$ in $2012, p=0.306$ ), and $32.8 \%$ (34.6\% in 2011 and $31.0 \%$ in $2012, p=0.004$ ), respectively. The distribution of CKD prevalence classified by the GFR and ACR categories is shown in Table 2. The prevalence of low, moderately increased, high, and very high CKD risk prognosis was $92.0 \%, 6.3 \%, 1.1 \%$, and $0.6 \%$, respectively. The prevalence of GFR $<60 \mathrm{~mL} / \mathrm{min} / 1.73 \mathrm{~m}^{2}$ was $2.2 \%$, whereas $\mathrm{ACR} \geq 30 \mathrm{mg} / \mathrm{g}$ was $6.5 \%$ (A2 of $5.6 \%$ and $\mathrm{A}_{3}$ of $\left.0.9 \%\right)$. The prevalence of $\mathrm{ACR} \geq 30 \mathrm{mg} / \mathrm{g}$ in individuals with GFR $\geq 60 \mathrm{~mL} / \mathrm{min} / 1.73 \mathrm{~m}^{2}$ was $5.7 \%$ (A2 of $5.1 \%$ and $A_{3}$ of $0.6 \%$ ).

\section{Comparison of GFR using CKD-EPI equations and the MDRD Study equation}

The prevalence of each estimated GFR category using

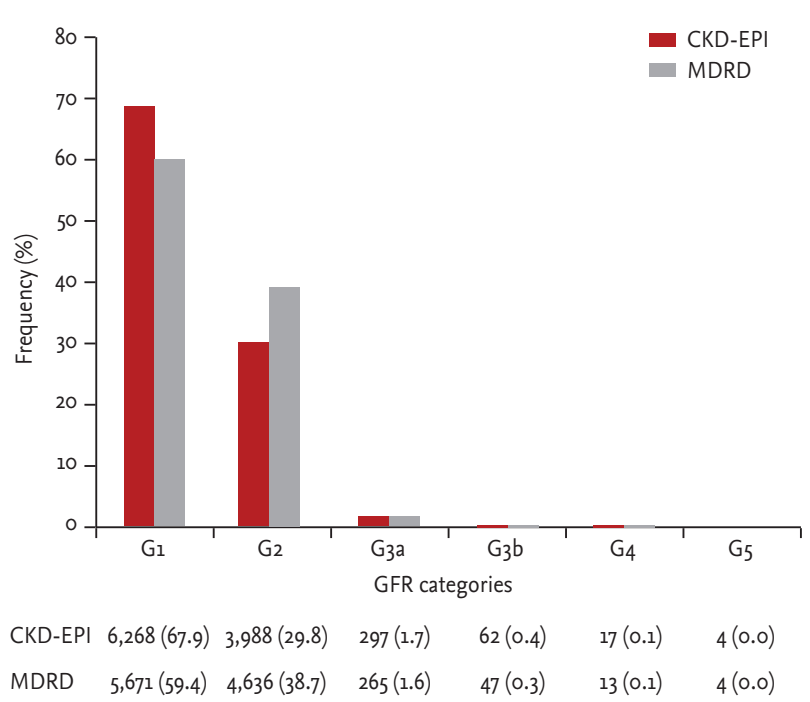

Figure 2. Comparison of glomerular filtration rate (GFR) prevalence estimated by using the Chronic Kidney Disease Epidemiology Collaboration equation (CKD-EPI) versus Modification of Diet in Renal Disease Study equation (MDRD) Study equations. Values are presented as number (\%) in the table. 
Table 1. Characteristics of the study population

\begin{tabular}{|c|c|c|c|c|}
\hline Characteristic & 2011 & 2012 & Total & $p$ value $^{a}$ \\
\hline Number & 5,388 & 5,248 & 10,636 & 0.087 \\
\hline Male sex & $2,431(52.5)$ & $2,327(52.7)$ & $4,758(52.6)$ & 0.839 \\
\hline Age, yr & $45.7(44.8-46.5)$ & $45 \cdot 9(45.2-46.6)$ & $45.8(45 \cdot 2-46.3)$ & 0.690 \\
\hline Body mass index $\geq 25$ & $1,759(32.8)$ & $1,699(33.6)$ & $3,458(33.2)$ & 0.577 \\
\hline Serum creatinine, mg/dL & $0.85(0.85-0.86)$ & $0.86(0.85-0.87)$ & $0.86(0.85-0.86)$ & 0.428 \\
\hline $\mathrm{GFR}, \mathrm{mL} / \mathrm{min} / 1.73 \mathrm{~m}^{2}$ & $97(96-97)$ & $96(95-97)$ & $96(96-97)$ & 0.452 \\
\hline Urine albumin, $\mu \mathrm{g} / \mathrm{mL}$ & $23(16-30)$ & $22(18-26)$ & $22(19-26)$ & 0.787 \\
\hline Urine creatinine, mg/dL & $163(159-167)$ & $168(164-171)$ & $165(163-168)$ & 0.126 \\
\hline $\mathrm{ACR}, \mathrm{mg} / \mathrm{g}$ & $18(14-22)$ & $17(14-21)$ & $18(15-21)$ & 0.760 \\
\hline CKD prevalence & $55^{2}(7.8)$ & $575(8.0)$ & $1,127(7 \cdot 9)$ & 0.770 \\
\hline $\mathrm{SBP}, \mathrm{mmHg}$ & $118(117-119)$ & $118(117-119)$ & $118(117-119)$ & 0.400 \\
\hline $\mathrm{DBP}, \mathrm{mmHg}$ & $76(76-77)$ & $76(76-77)$ & $76(76-77)$ & 0.761 \\
\hline HTN prevalence & $1,801(27.2)$ & $1,824(28.4)$ & $3,625(27.8)$ & 0.380 \\
\hline Hemoglobin Aıc, \% & $5 \cdot 7(5 \cdot 66-5,72)$ & $5.7(5.67-5.73)$ & $5.7(5.67-5.71)$ & 0.725 \\
\hline FBG, mg/dL & $97(96-98)$ & $97(97-98)$ & $97(97-98)$ & 0.271 \\
\hline DM prevalence & $651(9.7)$ & $643(9.0)$ & $1,294(9 \cdot 3)$ & 0.306 \\
\hline Total cholesterol, mg/dL & $190(188-191)$ & $189(188-191)$ & $190(188-191)$ & 0.906 \\
\hline HDL-C, mg/dL & $49(48-49)$ & $50(50-51)$ & $49(49-50)$ & $<0.001$ \\
\hline $\mathrm{LDL}-\mathrm{C}, \mathrm{mg} / \mathrm{dL}$ & $115(114-116)$ & $113(112-114)$ & $114(113-115)$ & 0.020 \\
\hline Triglycerides, mg/dL & $136(132-140)$ & $137(132-141)$ & $136(133-139)$ & 0.797 \\
\hline Dyslipidemia prevalence & $2,053(34.6)$ & $1,799(31.0)$ & $3,852(32.8)$ & 0.004 \\
\hline
\end{tabular}

Values are presented as number (\%) or mean ( $95 \%$ confidence interval).

GFR, estimated glomerular filtration rate; ACR, albumin-to-creatinine ratio; CKD, chronic kidney disease; SBP, systolic blood pressure; DBP, diastolic blood pressure; HTN, hypertension; FBG, fasting blood glucose; DM, diabetes mellitus; HDL-C, high density lipoprotein cholesterol; LDL-C, low density lipoprotein cholesterol.

${ }^{\mathrm{a} C o m p a r i s o n}$ between 2011 and 2012.

CKD-EPI equations and the MDRD Study equation is shown in Fig. 2. The prevalence of G1 was $67.9 \%$ and $59.4 \%$ by CKD-EPI equations and the MDRD study equation, and $\mathrm{G} 2$ was $29.8 \%$ and $38.7 \%$, respectively. The prevalence of GFR $<60 \mathrm{~mL} / \mathrm{min} / 1.73 \mathrm{~m}^{2}$ by the MDRD study equation was $2.0 \%$, which was significantly less than the $2.2 \%$ by CKD-EPI equations $(p<0.001)$.

\section{Comparison of ACR with proteinuria}

The prevalence of at least $1+$ proteinuria measured by test strip was $1.2 \%$. The frequency of ACR categories compared with proteinuria categories is shown in Table 3. The prevalence of at least $1+$ proteinuria in $A_{1}$, expected to be fully negative, was $0.1 \%$ whereas - or \pm proteinuria in $\mathrm{A}_{2}$ and $\mathrm{A}_{3}$, expected to be fully positive, were 5.3\% and $0.3 \%$, respectively.

\section{Associations of risk factors with CKD}

Some interactions between demographic covariates, such as gender, age, and BMI, were present. The risk of $\mathrm{BMI} \geq 25$ for men was higher than for women (OR, 1.296; 95\% CI, 1.164 to $1.443 ; p<0.001)$. The study population was stratified into six groups by age in 10-year increments: 19 to 28,29 to 38,39 to 48,49 to 58,59 to 68 , and $\geq$ 68. The ORs of increasing age (using the age group of 19 to 28 as a reference) to BMI $\geq 25$ were 1.731 (95\% CI, 1.400 to 2.139 ), 2.150 (95\% CI, 1.757 to 2.632 ), 1.689 (95\% CI, 1.376 to 2.074 ), 2.092 (95\% CI, 1.690 to 2.590 ), and 1.554 (95\% CI, 1.255 to 1.924; $p=0.001$ ), respectively. In addition, the composition of men significantly decreased in the $\geq 49$ age groups; ORs of men to increasing age (using the age group of 19 to 28 as a reference) were 0.931 (95\% CI, 0.784 to 1.105), 0.894 (95\% CI, 0.750 to 1.065), 0.735 (95\% CI, 0.625 to 0.864 ), 0.691 (95\% CI, 0.578 to 0.825 ), and 0.505 (95\% CI, 
Table 2. Prognosis of chronic kidney disease by GFR and ACR category among the Korean adult population

\begin{tabular}{|c|c|c|c|c|}
\hline \multirow{2}{*}{ Variable } & \multicolumn{3}{|c|}{ ACR, mg/g } & \multirow{2}{*}{ Total } \\
\hline & $\mathrm{A} 1(<30)$ & $\mathrm{A} 2(30-300)$ & $A_{3}(>300)$ & \\
\hline \multicolumn{5}{|c|}{ GFR, $\mathrm{mL} / \mathrm{min} / 1.73 \mathrm{~m}^{2}$} \\
\hline $\mathrm{G} 1(\geq 90)$ & $5,932(65.0)^{\mathrm{a}}$ & $302(2.7)^{b}$ & $34(0.2)^{c}$ & $6,268(67.9)$ \\
\hline $\mathrm{G} 2(60-89)$ & $3,577(27.0)^{\mathrm{a}}$ & $352(2.4)^{b}$ & $59(0.4)^{c}$ & $3,988(29.8)$ \\
\hline G3a $(45-59)$ & $217(1.2)^{\mathrm{b}}$ & $63(0.4)^{c}$ & $17(0.1)^{\mathrm{d}}$ & $297(1.7)$ \\
\hline $\mathrm{G}_{3} \mathrm{~b}(3 \mathrm{O}-44)$ & $25(0.1)^{c}$ & $21(0.1)^{\mathrm{d}}$ & $16(0.1)^{\mathrm{d}}$ & $62(0.4)$ \\
\hline $\mathrm{G}_{4}(15-29)$ & $5(0.0)^{d}$ & $6(0.0)^{\mathrm{d}}$ & $6(0.0)^{\mathrm{d}}$ & $17(0.1)$ \\
\hline $\mathrm{G}_{5}(<15)$ & $-^{\mathrm{d}}$ & $1(0.0)^{d}$ & $3(0.0)^{d}$ & $4(0.0)$ \\
\hline Total & $9,756(93.5)$ & $745(5.6)$ & $135(0.9)$ & $10,636(100.0)$ \\
\hline
\end{tabular}

Values are presented as number (\%).

GFR, glomerular filtration rate; ACR, albumin-to-creatinine ratio.

${ }^{a}$ Low risk (if no other markers of kidney disease, no chronic kidney disease).

${ }^{\mathrm{b}}$ Moderately increased risk.

${ }^{\mathrm{c}}$ High risk.

${ }^{\mathrm{d}}$ Very high risk.

Table 3. Comparison of urine protein excretion between ACR and proteinuria measured by test strip

\begin{tabular}{|c|c|c|c|c|}
\hline \multirow{2}{*}{ Variable } & \multicolumn{3}{|c|}{ ACR } & \multirow{2}{*}{ Total } \\
\hline & $\mathrm{A}_{1}$ & $\mathrm{~A} 2$ & $\mathrm{~A}_{3}$ & \\
\hline \multicolumn{5}{|c|}{ Proteinuria } \\
\hline- & $9,122(86.4)$ & $508(3.6)$ & $17(0.1)$ & 9,647 (90.1) \\
\hline \pm & $624(6.9)$ & $202(1.7)$ & $25(0.2)$ & $851(8.7)$ \\
\hline+ & $9(0.1)$ & $32(0.3)$ & $48(0.3)$ & $89(0.8)$ \\
\hline++ & o & $2(0.0)$ & $33(0.3)$ & $35(0.3)$ \\
\hline+++ & $1(0.0)$ & $1(0.0)$ & $12(0.1)$ & $14(0.1)$ \\
\hline Total & $9,756(93.5)$ & $745(5.6)$ & $135(0.9)$ & $10,636(100)$ \\
\hline
\end{tabular}

Values are presented as number (\%).

ACR, albumin-to-creatinine ratio.

0.421 to $0.607 ; p=0.001$ ), respectively. Thus, the association analysis was performed separately for each gender. Because CKD is defined by both GFR and albuminuria, the associations between the risk factors and either decreased GFR or increased ACR were also studied. The frequency of women among CKD patients was 54.3\%, which was significantly higher than that for men $(p<$ 0.001). Among individuals with ACR $\geq 30 \mathrm{mg} / \mathrm{g}$, women accounted for $55.4 \%$ ( $p<0.001)$; however, women constituted $48.7 \%$ of GFR $<60 \mathrm{~mL} / \mathrm{min} / 1.73 \mathrm{~m}^{2}(p=0.683)$. The ORs of gender to CKD, ACR $\geq 30 \mathrm{mg} / \mathrm{g}$, and GFR $<60$ $\mathrm{mL} / \mathrm{min} / 1.73 \mathrm{~m}^{2}$ for men were 1.354 (95\% CI, 1.162 to 1.577; $p<0.001$ ), 1.416 (95\% CI, 1.193 to $1.682 ; p<0.001$ ), and 1.057 (95\% CI, 0.811 to $1.376 ; p=0.683$ ), respectively. The preva- lence of BMI $\geq 25$, CKD, GFR $<60 \mathrm{~mL} / \mathrm{min} / 1.73 \mathrm{~m}^{2}$, ACR $\geq 30 \mathrm{mg} / \mathrm{g}$, DM, hypertension, and dyslipidemia stratified by age categories in men and women are shown in Tables 4 and 5. The ORs of covariates to the prevalence of CKD, GFR $<60 \mathrm{~mL} / \mathrm{min} / 1.73 \mathrm{~m}^{2}$, and $\mathrm{ACR} \geq 30 \mathrm{mg} / \mathrm{g}$, after adjusting for age, BMI, hypertension, DM, and dyslipidemia in men and women are listed in Table 6.

Hypertensive patients showed a higher risk of CKD than the normotensive population (OR, 3.394 for men; OR, 2.935 for women; both $p<0.0001$ ). The ORs of hypertension to cause albuminuria were $3.351(p<0.001)$ for men and $3.067(p<0.001)$ for women, and to decrease GFR, $3.436(p<0.001)$ for men and $1.904(p=0.006)$ for women. Diabetic patients showed a higher risk of CKD 
Table 4. Prevalence of CKD, GFR, and ACR, stratified by age groups, in men

\begin{tabular}{|c|c|c|c|c|c|c|c|c|}
\hline Age, yr & Male sex & $\mathrm{BMI} \geq 25$ & CKD & $\begin{array}{c}\text { GFR, }<60 \mathrm{~mL} / \\
\min / 1.73 \mathrm{~m}^{2}\end{array}$ & $\mathrm{ACR} \geq 30 \mathrm{mg} / \mathrm{g}$ & Diabetes mellitus & Hypertension & Dyslipidemia \\
\hline $19-28$ & $471(18.1)$ & $130(5.0)$ & $5(0.2)$ & - & $5(0.2)$ & $1(0.0)$ & $46(1.8)$ & $98(3.8)$ \\
\hline $29-38$ & $786(21.2)$ & $318(8.8)$ & $20(0.5)$ & - & $20(0.5)$ & $18(0.6)$ & $131(3 \cdot 5)$ & $266(6.9)$ \\
\hline $39-48$ & $858(22.4)$ & $364(9.9)$ & $39(1.0)$ & $1(0.0)$ & $38(1.0)$ & $61(1.5)$ & $235(6.3)$ & $340(8.4)$ \\
\hline $49-58$ & $927(19.5)$ & $320(6.4)$ & $76(1.5)$ & $19(0.4)$ & $68(1.3)$ & $138(2.8)$ & $350(7 \cdot 4)$ & $409(8.5)$ \\
\hline $59-68$ & $859(10.7)$ & $289(3.6)$ & $128(1.6)$ & $37(0.5)$ & $105(1.3)$ & $216(2.7)$ & $468(5.8)$ & $399(4.7)$ \\
\hline$\geq 68$ & $857(8.1)$ & $218(2.1)$ & $220(2.1)$ & $131(1.2)$ & $132(1.3)$ & $206(2.0)$ & $513(4.9)$ & $379(3.4)$ \\
\hline Total & $4,758(100)$ & $1,639(35.9)$ & $488(6.9)$ & $188(2.2)$ & $368(5.5)$ & $640(9.6)$ & $1,743(29.7)$ & $1,891(35.8)$ \\
\hline
\end{tabular}

Values are presented as number (\%).

CKD, chronic kidney disease; GFR, glomerular filtration rate; ACR, albumin-to-creatinine ratio; BMI, body mass index.

Table 5. Prevalence of CKD, GFR, and ACR, stratified by age groups, in women

\begin{tabular}{lcccccccc}
\hline Age, yr & Female sex & BMI $\geq 25$ & CKD & $\begin{array}{c}\text { GFR, }<60 \mathrm{~mL} / \\
\mathrm{min} / 1.73 \mathrm{~m}^{2}\end{array}$ & ACR $\geq 30 \mathrm{mg} / \mathrm{g}$ & $\begin{array}{l}\text { Diabetes } \\
\text { mellitus }\end{array}$ & Hypertension & Dyslipidemia \\
\hline $19-28$ & $518(14.6)$ & $75(2.2)$ & $18(0.4)$ & - & $18(0.4)$ & $2(0.1)$ & $3(0.1)$ & $43(1.3)$ \\
$29-38$ & $931(18.4)$ & $190(4.4)$ & $27(0.6)$ & $1(0.0)$ & $27(0.6)$ & $17(0.3)$ & $29(0.7)$ & $131(2.8)$ \\
$39-48$ & $987(20.2)$ & $291(6.5)$ & $53(1.0)$ & $2(0.1)$ & $53(1.0)$ & $41(0.9)$ & $119(2.8)$ & $212(4.7)$ \\
$49-58$ & $1,252(21.4)$ & $422(7.2)$ & $87(1.5)$ & $7(0.1)$ & $85(1.5)$ & $121(2.1)$ & $375(6.2)$ & $486(8.0)$ \\
$59-68$ & $1,065(12.5)$ & $436(5.3)$ & $142(1.7)$ & $26(0.3)$ & $128(1.6)$ & $200(2.3)$ & $555(6.5)$ & $554(6.5)$ \\
$\geq 68$ & $1,125(13.0)$ & $405(4.6)$ & $312(3.8)$ & $156(1.8)$ & $201(2.5)$ & $273(3.3)$ & $801(9.4)$ & $535(6.1)$ \\
Total & $5,878(100)$ & $1,819(30.2)$ & $639(9.1)$ & $192(2.3)$ & $512(7.7)$ & $654(9.1)$ & $1,882(25.7)$ & $1,0.961(29.4)$ \\
\hline
\end{tabular}

Values are presented as number (\%).

CKD, chronic kidney disease; GFR, glomerular filtration rate; ACR, albumin-to-creatinine ratio; BMI, body mass index.

than the nondiabetic population (OR, 3.145 for men; OR, 1.983 for women; both $p<0.001)$. The ORs of DM to cause albuminuria were $3.144(p<0.001)$ for men and $2.022(p<0.001)$ for women, and to decrease GFR 1.965 $(p=0.004)$ for men and $1.764(p=0.013)$ for women. Dyslipidemia showed a significant association with CKD in women (OR, 1.324; $p=0.023)$, but not in men (OR, 1.175; $p$ $=0.243)$. Increasing age and obesity $(\mathrm{BMI} \geq 25)$ showed a positive correlation with CKD.

\section{DISCUSSION}

This study is the first to report CKD prevalence using GFR estimated by using CKD-EPI equations together with ACR for albuminuria in the Korean population. The prevalence of CKD was 7.9\% in the KNHANES samples of 2011 and 2012 in Korean adults aged $\geq 19$, and the CKD risk prognosis was reported in Table 2. The
CKD risk prognosis from moderately increased to very high reported in this study was lower than that in the USA [15]. GFR and albuminuria are independent and complementary predictors of important clinical outcomes, including CKD progression, end-stage renal disease, acute kidney injury, cardiovascular mortality, and all-cause mortality [16-19]. The frequencies of GFR $<60$ $\mathrm{mL} / \mathrm{min} / 1.73 \mathrm{~m}^{2}$ showed sharp increases starting from around age 50 for men and 60 for women, whereas those of ACR $\geq 30 \mathrm{mg} / \mathrm{g}$ increased relatively gradually, starting from earlier ages, as shown in Table 4 and 5. The rate of decline of measured GFR was reported to double in kidney transplantation donors aged $>45$ years [20]. The prevalence of estimated GFR $<60 \mathrm{~mL} / \mathrm{min} / 1.73 \mathrm{~m}^{2}$ using CKD-EPI equations and the MDRD Study equation was $2.2 \%$ and $2.0 \%$ in this study, but $2.6 \%$ and $3.2 \%$, respectively in the previous study [7]. The statistical method used in the current study was complex sample analysis incorporating sampling weight, in contrast to the previ- 
Table 6. Results for multivariate logistic regression analysis to examine the associations between covariates and CKD, GFR, and ACR by gender

\begin{tabular}{|c|c|c|c|c|c|c|}
\hline \multirow{2}{*}{ Covariates } & \multicolumn{2}{|l|}{ CKD } & \multicolumn{2}{|c|}{$\mathrm{GFR}<60 \mathrm{~mL} / \mathrm{min} / 1.73 \mathrm{~m}^{2}$} & \multicolumn{2}{|c|}{$\mathrm{ACR} \geq 30 \mathrm{mg} / \mathrm{g}$} \\
\hline & OR $(95 \% \mathrm{CI})$ & $p$ value & $\mathrm{OR}(95 \% \mathrm{CI})$ & $p$ value & OR $(95 \% \mathrm{CI})$ & $p$ value \\
\hline \multicolumn{7}{|l|}{ Male } \\
\hline Age & & $<0.001$ & & $<0.001$ & & $<0.001$ \\
\hline $19-28$ & $0.068(0.021-0.221)$ & & - & & $0.134(0.041-0.436)$ & \\
\hline $29-38$ & $0.134(0.074-0.243)$ & & - & & $0.268(0.144-0.499)$ & \\
\hline $39-48$ & $0.209(0.130-0.335)$ & & $0.009(0.001-0.064)$ & & $0.410(0.252-0.666)$ & \\
\hline $49-58$ & $0.308(0.217-0.437)$ & & $0.156(0.089-0.272)$ & & $0.519(0.353-0.764)$ & \\
\hline $59-68$ & $0.474(0.342-0.657)$ & & $0.237(0.157-0.359)$ & & $0.723(0.505-1.036)$ & \\
\hline$\geq 68$ & 1 & & 1 & & 1 & \\
\hline $\mathrm{BMI} \geq 25$ & $1.514(1.117-2.051)$ & 0.008 & $1.521(1.006-2.298)$ & 0.047 & $1.407(1.012-1.956)$ & 0.042 \\
\hline Diabetes mellitus & $3.145(2.318-4.267)$ & $<0.001$ & $1.965(1.244-3.104)$ & 0.004 & $3.144(2.233-4.426)$ & $<0.001$ \\
\hline Hypertension & $3.394(2.473-4.658)$ & $<0.001$ & $3.436(2.172-5.435)$ & $<0.001$ & $3.351(2.345-4.788)$ & $<0.001$ \\
\hline Dyslipidemia & $1.175(0.896-1.541)$ & 0.243 & $1.544(1.038-2.296)$ & 0.032 & $1.103(0.809-1.504)$ & 0.534 \\
\hline \multicolumn{7}{|l|}{ Female } \\
\hline Age & & $<0.001$ & & $<0.001$ & & 0.004 \\
\hline $19-28$ & $0.256(0.141-0.467)$ & & - & & $0.465(0.250-0.865)$ & \\
\hline $29-38$ & $0.224(0.127-0.397)$ & & $0.010(0.001-0.074)$ & & $0.408(0.227-0.733)$ & \\
\hline $39-48$ & $0.287(0.187-0.438)$ & & $0.034(0.007-0.151)$ & & $0.520(0.341-0.792)$ & \\
\hline $49-58$ & $0.305(0.221-0.419)$ & & $0.040(0.017-0.096)$ & & $0.541(0.382-0.765)$ & \\
\hline $59-68$ & $0.427(0.322-0.567)$ & & $0.179(0.104-0.308)$ & & $0.685(0.504-0.929)$ & \\
\hline$\geq 68$ & 1 & & 1 & & 1 & \\
\hline $\mathrm{BMI} \geq 25$ & $1.630(1.303-2.039)$ & $<0.001$ & $1.376(1.008-1.879)$ & 0.045 & $1.612(1.256-2.067)$ & $<0.001$ \\
\hline Diabetes mellitus & $1.983(1.480-2.657)$ & $<0.001$ & $1.764(1.129-2.756)$ & 0.013 & $2.022(1.505-2.717)$ & $<0.001$ \\
\hline Hypertension & $2.935(2.153-4.002)$ & $<0.001$ & $1.904(1.204-3.010)$ & 0.006 & $3.067(2.187-4.300)$ & $<0.001$ \\
\hline Dyslipidemia & $1.324(1.040-1.686)$ & 0.023 & $1.361(0.891-2.079)$ & 0.153 & $1.254(0.977-1.610)$ & 0.075 \\
\hline
\end{tabular}

CKD, chronic kidney disease; GFR, glomerular filtration rate; ACR, albumin-to-creatinine ratio; OR, odds ratio; CI, confidence interval; BMI, body mass index.

ous study which did not consider weight in the analysis. The frequencies of GFR stage $\mathrm{G}_{1}$ and $\mathrm{G} 2$ by CKD-EPI equations were $67.7 \%$ and $29.8 \%$, whereas those by the MDRD Study equation were $59.4 \%$ and $38.7 \%$, respectively, in this study. These numbers are comparable to the previous study, considering the different analytical methods; the frequencies of GFR stage $\mathrm{G}_{1}$ and $\mathrm{G} 2$ by CKD-EPI equations were $64.5 \%$ and $28.9 \%$, whereas those by the MDRD Study equation were $47.6 \%$ and $49.2 \%$, respectively [7]. These results confirm the tendency to underestimate GFR by the MDRD Study equation when GFR is $60 \mathrm{~mL} / \mathrm{min} / 1.73 \mathrm{~m}^{2}$ or greater. The previous report of lower prevalence of GFR stage G3 using CKDEPI equations than with the MDRD Study equation was not observed in this study [5]. Among the population of the previous study, the frequencies of $\mathrm{G}_{3}$ by CKD-EPI equations and the MDRD Study equation (33.2\% and $37.2 \%$, respectively) were far greater than those among the Korean population (2.1\% and $1.9 \%$, respectively). This difference in the study population might explain the current result.

The prevalence of ACR $\geq 30 \mathrm{mg} / \mathrm{g}$ in the entire study population, and ACR $\geq 30 \mathrm{mg} / \mathrm{g}$ in individuals with GFR $\geq 60 \mathrm{~mL} / \mathrm{min} / 1.73 \mathrm{~m}^{2}$ was $6.5 \%$ and $5.7 \%$, respectively, in this study. The prevalence of ACR $\geq 30 \mathrm{mg} / \mathrm{g}$ in the entire study population and ACR $\geq 30 \mathrm{mg} / \mathrm{g}$ in individuals with $\mathrm{GFR} \geq 60 \mathrm{~mL} / \mathrm{min} / 1.73 \mathrm{~m}^{2}$ aged $\geq 35$ years was $10.2 \%$ and $8.7 \%$, respectively, in the previous Korean popula- 
tion [11]. Besides the different age criteria, the populations of the previous and current study are quite different; the previous study was designed by the researcher group considering age, gender, and city factors not using the KNHANES sample data. Although the absolute values are different, the important point in common is that a large number of people have albuminuria before GFR decreases to $<60 \mathrm{~mL} / \mathrm{min} / 1.73 \mathrm{~m}^{2}(85.3 \%$ and $87.7 \%$ of the albuminuria cases did not have decreased GFR in the previous and current study, respectively). As shown in Table 3, test strips did not detect albuminuria in $81.5 \%$ of cases. At present, laboratory tests for albuminuria are not routinely performed during regular physical examinations in Korea. This might result in the failure of early detection of kidney disorders.

The prevalence of CKD in men and women was $6.9 \%$ and $9.1 \%$, respectively $(p<0.001)$. Besides gender itself, age distribution, frequency of obesity (BMI $\geq 25$ ), and concurrent chronic diseases, including DM, hypertension, and dyslipidemia, were different according to gender. Thus, the risk of CKD is higher in women than men (OR, 1.354; $p<0.001)$, and the difference seems to be caused by the susceptibility to albuminuria (OR, 1.416; $p<0.001)$ rather than decreased GFR (OR, 1.057; $p=$ 0.683). When GFR was estimated using the MDRD Study equation, the increased risks of $\mathrm{GFR}<60 \mathrm{~mL} / \mathrm{min} / 1.73$ $\mathrm{m}^{2}$ in women were observed in previous studies: OR, 3.16 ( $p<0.001)$ using KNHANES III and IV-1 [12]; frequencies of $3.4 \%, 9.7 \%, 10.2 \%$, and $4.6 \%$ in women, and $1.0 \%, 5.4 \%, 3.1 \%$, and $2.6 \%$ were seen in men through KNHANES I-IV [13]. There seemed to be no difference in ACR according to gender in the previous study (10.1\% and $10.3 \%$ in women and men, respectively) [11]. A similar observation was reported for proteinuria by gender (frequencies of 3.3\%, 2.3\%, 1.4\%, and 1.9\% in women, and $3.1 \%, 3.0 \%, 2.8 \%$, and $2.2 \%$ in men through KNHANES I to IV) [13]. Direct comparison is difficult due to the difference in the designs of study populations [11] and laboratory test methods using dipstick proteinuria and ACR [13]. Increasing age and obesity (BMI $\geq 25)$ correlated significantly with CKD, as shown in Table 6, which agreed with previous studies [11-13]. Hypertension and $\mathrm{DM}$ are both major risk factors and consequences of CKD [21-23]. Concurrent chronic diseases such as DM and hypertension increased the risk of CKD consistently [11-13]. Diabetic patients are susceptible to glomerular disease and hypertensive patients to renal vascular disease [8]. Since the prevalence of DM was higher in men than women, with the opposite for prevalence of CKD, the OR of DM to CKD in men was higher than women, as shown in Table 6. Moreover, DM was correlated with increased ACR rather than decreased GFR in this study. Since increased ACR appeared at an earlier age in women than men, and decreased GFR was preceded by increased ACR, the difference between ORs to GFR $<60 \mathrm{~mL} / \mathrm{min} / 1.73 \mathrm{~m}^{2}$ and ACR $\geq 30 \mathrm{mg} / \mathrm{g}$ in hypertensive women was greater than hypertensive men. On the other hand, the association of CKD with dyslipidemia has not yet been clarified. Limited availability of direct LDL-C measurement, unstandardized laboratory assay methods for HDL-C, and lack of a uniform definition of dyslipidemia between the studies made it difficult to evaluate their associations.

The major limitation of this study is the lack of the evaluation for chronicity assessment and use of single measurements for laboratory tests due to the cross-sectional data characteristics. According to the definition of CKD by the KDIGO guideline, the functional or structural disorder should last $>3$ months. The rationale for defining chronicity is to differentiate CKD from acute kidney diseases, which may require different interventions and have different etiologies and outcomes [24].

In addition, there is still the discrepancy between measured and estimated GFR using CKD-EPI equations. GFR estimation using equations is convenient, but measurement of GFR should be considered to diagnose a specific patient. Finally, association analysis could not provide conclusive causal relationships due to the study characteristics. However, the representative features of the KNHANES sample data cannot be overlooked.

In conclusion, the prevalence of CKD using estimated GFR by the CKD-EPI equation and ACR for albuminuria measurement was $7.9 \%$ in the KNHANES samples of 2011 and 2012 in Korean adults aged $\geq 19$. The prevalence of albuminuria (ACR $\geq 30 \mathrm{mg} / \mathrm{g}$ ) before decline in GFR (GFR $\left.\geq 60 \mathrm{~mL} / \mathrm{min} / 1.73 \mathrm{~m}^{2}\right)$ was $5.7 \%$. Despite KDIGO guidelines to prevent or delay progression of CKD in its early stages, implementation remains difficult due to practical limitations [25]. The failure to recognize CKD in its early stage may result in complications such as premature RRT. Thus, urinalysis to detect albumin should be strongly recommended for patients with 
higher risk of CKD and CVD $[8,26]$. As there is a consensus about the risk of CKD with DM and hypertension, regular albuminuria testing for at least these patients to detect kidney disease at an early stage must be considered. Public policies for effective delivery of appropriate CKD care should be addressed.

\section{KEY MESSAGE}

1. The prevalence of chronic kidney disease (CKD) was estimated to be $7.9 \%$ in the Korea National Health and Nutrition Examination Survey samples of 2011 and 2012 in Korean adults aged $\geq 19$.

2. Early identification of CKD paired with appropriate management and earlier referral to specialty kidney services results in economic and clinical benefits.

3. The majority of CKD patients have albuminuria prior to a decrease in glomerular filtration rate.

4. Regular laboratory tests for albuminuria in the high-risk group, especially for hypertensive or diabetes mellitus patients, should contribute to early detection of CKD.

\section{Conflict of interest}

No potential conflict of interest relevant to this article was reported.

\section{Acknowledgments}

This work was supported by Gachon Institute of Pharmaceutical Sciences Research Fund 2014.

\section{REFERENCES}

1. National Kidney Foundation. K/DOQI clinical practice guidelines for chronic kidney disease: evaluation, classification, and stratification. Am J Kidney Dis 2002;39(2 Suppl 1):S1-S266.

2. Eckardt KU, Coresh J, Devuyst O, et al. Evolving importance of kidney disease: from subspecialty to global health burden. Lancet 2013;382:158-169.

3. James MT, Hemmelgarn BR, Tonelli M. Early recognition and prevention of chronic kidney disease. Lancet 2010;375:1296-1309.
4. Levey AS, Coresh J, Greene T, et al. Using standardized serum creatinine values in the modification of diet in renal disease study equation for estimating glomerular filtration rate. Ann Intern Med 2006;145:247-254.

5. Levey AS, Stevens LA, Schmid CH, et al. A new equation to estimate glomerular filtration rate. Ann Intern Med 2009;150:604-612.

6. Stevens LA, Coresh J, Feldman HI, et al. Evaluation of the modification of diet in renal disease study equation in a large diverse population. J Am Soc Nephrol 2007;18:27492757.

7. Jeong TD, Lee W, Chun S, et al. Comparison of the MDRD study and CKD-EPI equations for the estimation of the glomerular filtration rate in the Korean general population: the fifth Korea National Health and Nutrition Examination Survey (KNHANES V-1), 2010. Kidney Blood Press Res 2013;37:443-450.

8. Levin A, Stevens PE, Bilous RW, et al. Kidney disease: improving global outcomes (KDIGO) CKD work group. KDIGO 2012 clinical practice guideline for the evaluation and management of chronic kidney disease. Kidney Int Suppl 2013;3:1-150.

9. Levey AS, Becker C, Inker LA. Glomerular filtration rate and albuminuria for detection and staging of acute and chronic kidney disease in adults: a systematic review. JAMA 2015;313:837-846.

10. Jin DC. Major changes and improvements of dialysis therapy in Korea: review of end-stage renal disease registry. Korean J Intern Med 2015;30:17-22.

11. Kim S, Lim CS, Han DC, et al. The prevalence of chronic kidney disease (CKD) and the associated factors to CKD in urban Korea: a population-based cross-sectional epidemiologic study. J Korean Med Sci 2009;24 Suppl:S11-S21.

12. Lee SW, Kim YC, Oh SW, et al. Trends in the prevalence of chronic kidney disease, other chronic diseases and health-related behaviors in an adult Korean population: data from the Korean National Health and Nutrition Examination Survey (KNHANES). Nephrol Dial Transplant 2011;26:3975-3980.

13. Kang HT, Lee J, Linton JA, Park BJ, Lee YJ. Trends in the prevalence of chronic kidney disease in Korean adults: the Korean National Health and Nutrition Examination Survey from 1998 to 2009. Nephrol Dial Transplant 2013;28:927-936.

14. Korea Centers for Disease Control and Prevention. The fifth Korea National Health and Nutrition Examination 
Survey (KNHANES V) 2010. Cheongju (KR): Korea Centers for Disease Control and Prevention, 2010 [cited 2016 Jan 4]. Available from: http://knhanes.cdc.go.kr.

15. Levey AS, Coresh J. Chronic kidney disease. Lancet 2012;379:165-180.

16. Chronic Kidney Disease Prognosis Consortium, Matsushita K, van der Velde M, et al. Association of estimated glomerular filtration rate and albuminuria with all-cause and cardiovascular mortality in general population cohorts: a collaborative meta-analysis. Lancet 2010;375:20732081.

17. van der Velde M, Matsushita K, Coresh J, et al. Lower estimated glomerular filtration rate and higher albuminuria are associated with all-cause and cardiovascular mortality: a collaborative meta-analysis of high-risk population cohorts. Kidney Int 2011;79:1341-1352.

18. Astor BC, Matsushita K, Gansevoort RT, et al. Lower estimated glomerular filtration rate and higher albuminuria are associated with mortality and end-stage renal disease: a collaborative meta-analysis of kidney disease population cohorts. Kidney Int 2011;79:1331-1340.

19. Gansevoort RT, Matsushita K, van der Velde M, et al. Lower estimated GFR and higher albuminuria are associated with adverse kidney outcomes: a collaborative meta-analysis of general and high-risk population cohorts. Kidney Int 2011;80:93-104.
20. Poggio ED, Rule AD, Tanchanco R, et al. Demographic and clinical characteristics associated with glomerular filtration rates in living kidney donors. Kidney Int 2009;75:1079-1087.

21. O'Seaghdha CM, Perkovic V, Lam TH, et al. Blood pressure is a major risk factor for renal death: an analysis of 560352 participants from the Asia-Pacific region. Hypertension 2009;54:509-515.

22. Yamagata K, Ishida K, Sairenchi T, et al. Risk factors for chronic kidney disease in a community-based population: a 10-year follow-up study. Kidney Int 2007;71:159166.

23. Iseki K, Oshiro S, Tozawa M, Ikemiya Y, Fukiyama K, Takishita S. Prevalence and correlates of diabetes mellitus in a screened cohort in Okinawa, Japan. Hypertens Res 2002;25:185-190.

24. KDIGO AKI Work Group. KDIGO clinical practice guideline for acute kidney injury. Kidney Int Suppl 2012;2:1-138.

25. Rettig RA, Norris K, Nissenson AR. Chronic kidney disease in the United States: a public policy imperative. Clin J Am Soc Nephrol 2008;3:1902-1910.

26. Greenland P, Alpert JS, Beller GA, et al. 2010 ACCF/AHA guideline for assessment of cardiovascular risk in asymptomatic adults: a report of the American College of Cardiology Foundation/American Heart Association Task Force on Practice Guidelines. Circulation 2010;122:e584-e636. 